\title{
Mobilidade, participação e dados: o caso da aplicação do Waze for Cities Data na cidade de Joinville (SC)
}

Mobility, participation and data: the case study of Waze for Cities Data in Joinville (SC)

Stella Marina Yurí Hiroki [a] [D

[a] Pontifícia Universidade Católica de São Paulo (PUC-SP), São Paulo, SP, Brasil

Como citar: Hiroki, S. M. Y. (2021). Mobilidade, participação e dados: o caso da aplicação do Waze for Cities Data na cidade de Joinville (SC). urbe. Revista Brasileira de Gestão Urbana, v.13, e20200030. https://doi.org/10.1590/21753369.013.e20200030

\section{Resumo}

Este artigo descreve a metodologia do projeto Smart Mobility, responsável pela implementação do programa Waze for Cities Data. Desenvolvida pela Secretaria de Planejamento Urbano e Desenvolvimento Sustentável (Sepud) da cidade de Joinville (SC), tal metodologia tem seu planejamento de mobilidade ancorado em dados sobre o trânsito coletados pela empresa Waze. A partir do conceito de Cidades Inteligentes, a fundamentação teórica analisa a aplicação de Big Data no planejamento urbano orientado a dados, assim como a importância da participação dos cidadãos em projetos de tecnologia. Este estudo também aborda o histórico do aplicativo Waze e do programa de compartilhamento de dados bidirecional Waze for Cities Data. Trata-se de uma análise exploratória de estudo de caso, de caráter qualitativo, cujo instrumento de coleta foi a observação sistemática/não participante. Os resultados demonstram que a utilização dos dados coletados com o auxílio de plataformas de tecnologia pode contribuir para a otimização da gestão urbana, por meio de soluções mais próximas da realidade da população, gerando benefícios em tempo e produtividade. 0 projeto também posicionou o Brasil como referência mundial na adoção de dados coletados pelo Waze, reforçando, assim, a necessidade de iniciativas semelhantes, que sejam menos tecnocráticas e mais centradas no cidadão.

Palavras-chave: Cidades Inteligentes. Waze. Dados. Mobilidade. Participação.

\section{Abstract}

This article describes Smart Mobility's methodology, a project responsible for the implementation of Waze for Cities Data. Developed by the Secretaria de Planejamento Urbano e Desenvolvimento Sustentável (Sepud) of the city of Joinville (SC), the project's methodology has its mobility plan based on traffic data collected and provided by Waze. Grounded in the concept of Smart Cities, the study's theoretical framework analyzes the application of Big Data in data-driven urban planning, as well as the importance of citizen's participation in technology projects. When it comes to the study's methodology, a qualitative exploratory case study is presented, in which a

SMYH é pesquisadora sobre Cidades Inteligentes, doutora em Tecnologias da Inteligência e Design Digital (TIDD), e-mail: stellahiroki@gmail.com 
systematic/non-participant observation was used as data collection instrument. The study also presents a brief historical background of Waze and the bidirectional data sharing program Waze for Cities Data. Results demonstrate that the use of data collected by technology platforms can contribute to the optimization of urban management through solutions that are closer to the population's reality; what could generate benefits in time and productivity. The project also positioned Brazil as a world reference in the adoption of data collected by Waze, reinforcing, then, the need of similar initiatives that are less technocratic and more citizen-centered.

Keywords: Smart Cities. Waze. Data. Mobility. Participation.

\section{Introdução}

A partir da década de 1980, com a mudança no paradigma do gerenciamento das cidades para um modelo de empresariamento urbano (Harvey, 1996), a iniciativa privada identificou nas cidades um potencial para investimentos. Junto a esse cenário, o advento das Tecnologias da Informação e Comunicação (TICs) e a expansão da utilização da internet, na virada do século XXI, contribuíram para intensificar a "transformação dos sistemas de infraestrutura existentes em plataformas multifuncionais de informação e serviços" (Alvarez, 2017, p. 12). Ao mesmo tempo que, dentre os vários setores da gestão urbana, a mobilidade mostra-se como uma das áreas mais sensíveis a essas mudanças tecnológicas (Firmino \& Simão, 2019).

Nesse sentido, como explica De Waal \& Dignum (2017), a Cidade Inteligente pode ser compreendida como um conjunto de grupos de atores humanos e não humanos que, juntos, moldam as atuais práticas urbanas. Por isso, de acordo com Kitchin (2014), os defensores do movimento de Cidades Inteligentes argumentam que os dados permitem uma análise em tempo real da vida da cidade, novos modos de governança urbana, ao mesmo tempo em que fornecem a matéria-prima para imaginar e implementar, de forma mais eficiente e sustentável, cidades competitivas, produtivas, abertas e transparentes. "Os dados são, portanto, vistos como materiais constituintes essenciais para a concretização de uma visão de Cidade Inteligente" (Kitchin, 2014, p. 3).

Se, por um lado, não há uma única definição para Big Data, por outro, há um consenso entre os autores sobre o modelo dos 3 V's: volume, velocidade e variedade (Batty, 2016; Kitchin, 2017; Cunha et al., 2020). Volume se refere à enorme quantidade de dados a serem processados em terabytes ou petabytes (Kitchin, 2014), a velocidade, por sua vez, está relacionada ao rápido processamento entre a sua geração, e o seu uso e variedade indicam a grande diversidade de origem, formas e formatos dos dados, o que pode ser uma dificuldade para categorizar e tabular (Cunha et al., 2020). A esse modelo dos 3 V's, Batty (2016) complementa com outros 2; quais sejam: variabilidade e veracidade. 0 primeiro implica um grau de inconsistência em representação e o segundo na qualidade dos dados que têm impacto sobre a solidez da análise.

Para Batty (2016), o que torna o Big Data tão importante para o atual debate sobre o entendimento das cidades é a oportunidade de entender as mudanças a curto prazo. Por isso, o autor justifica que essa ação foi encapsulada pelo movimento das Cidades Inteligentes e o Big Data "revela tendências seculares que primeiro aparecem como pequenas mudanças na rotina, mas depois se manifestam em mudança de longo

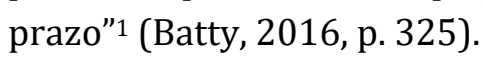

Dessa maneira, Kitchin (2016) explica que os sistemas de Big Data passaram a configurar e a definir a agenda urbana, de maneira a influenciar e controlar como os sistemas das cidades respondem e agem, resultando, desse modo, em uma nova forma de governança urbana altamente responsiva. Nessa perspectiva, os sistemas e as infraestruturas das cidades estão se tornando estreitamente interligados e integrados. Por isso, Benevolo et al. (2016) argumentam que a área de Smart Mobility (Mobilidade Inteligente) não é uma iniciativa única, mas um conjunto complexo de projetos e ações que, permeado pelas TICs, se organiza por meio de diferentes conteúdos e níveis de implementação tecnológica.

\footnotetext{
${ }^{1}$ Tradução da autora para: “(...) in revealing secular trends that first show up as small changes to the outine but then manifest themselves in longer term change" (Batty, 2016, p. 325).
} 
Em um estudo intitulado "Mobilidade inteligente: proposta de framework de Big Data analytics para análise de dados de mobilidade urbana em uma smart city", Buosi (2018) desenvolve uma pesquisa que, por meio de dados coletados a partir de entrevistas sobre o transporte público, propõe uma estrutura para analisar os dados da mobilidade urbana. 0 resultado obtido demonstrou a importância do engajamento da população para maior veracidade sobre os dados coletados, contudo, o estudo não explica como tal participação deve ser realizada.

Já no trabalho "Urbanismo movido a dados: o Big Data como ferramenta de gestão urbana", Menezes (2018) investiga, por meio de reportagens publicadas na imprensa sobre projetos urbanos orientados a dados, se estes seguem as definições de Big Data encontradas na literatura. No entanto, não há uma discussão sobre o método de aplicação dos dados coletados para uma solução no espaço urbano.

As pesquisas na literatura indicam o aumento do interesse dos estudos em analisar a influência do Big Data no planejamento das cidades. Entretanto, os trabalhos não aprofundam as discussões sobre a utilização de uma metodologia para a aplicação dos dados nos projetos urbanos, como também não abordam a participação da população. Levando-se em conta tais questões, o presente artigo busca, então, discutir a importância da utilização dos dados captados por meio dos aplicativos das empresas de mobilidade e que podem ser traduzidos com a colaboração de uma metodologia em benefício do espaço urbano.

Nesse sentido, o estudo de caso do projeto Waze for Cities Data na cidade de Joinville (SC), a ser aqui apresentado, objetiva analisar como as informações coletadas pela empresa Waze podem beneficiar a produtividade das pessoas e da cidade, a trajetória no fluxo de pedestres, motoristas, motociclistas e ciclistas e o incentivo à maior participação da população nas decisões dos gestores.

Com base em artigos e na descrição do estudo de caso, esta pesquisa aponta, para as cidades brasileiras, oportunidades para a implementação de projetos, tais como o da Cidade Inteligente, por meio de dados coletados por softwares que já integram o espaço urbano. Ao mesmo tempo, este estudo discute os desafios colocados pelas questões sociais e governamentais de um município na implementação de novas práticas como as propostas pelo Waze for Cities Data.

\section{Os níveis de participação dos cidadãos nos projetos de Cidades Inteligentes}

Os discursos e as práticas das Cidades Inteligentes não refletem apenas as noções já implícitas de cidadania, mas também adicionam ao repertório do espaço urbano novos exercícios para a participação dos cidadãos. De Waal \& Dignum (2017) explicam que esse movimento acontece por meio dos modelos organizacionais e interações aplicadas pelas tecnologias que as Cidades Inteligentes oferecem.

Desse modo, os autores elucidam sobre três conjuntos que emergiram e se sobrepõem parcialmente sobre a relação entre a cidadania e as Cidades Inteligentes; são eles: 1. a Sala de Controle, que concebe a cidade como um conjunto de infraestruturas e serviços; 2. A Cidade Criativa, que vislumbra a cidade pelo prisma da economia e pondera sobre os sistemas locais e regionais de inovação e; 3.0 discurso dos Cidadãos Inteligentes (Smart Citizens), que aborda a cidade como uma comunidade política e cívica. A partir desses três grupos, os autores afirmam que "[a]s infraestruturas digitais, como as Cidades Inteligentes, podem constituir tanto novos atos de cidadania, como um conjunto mais amplo de processos que constituem os pontos de partida da cultura cívica" (De Waal \& Dignum, 2017, p. 264).

Consonante aos três grupos indicados por De Wall \& Dignum (2017), Cardullo \& Kitchin (2019) asseveram que as empresas e cidades passaram a reformular suas iniciativas para serem centradas no cidadão, com o objetivo de responderem às críticas de que a Cidade Inteligente era demasiadamente tecnocrática e instrumental. Desse modo, empresas como IBM e Cisco adicionaram a seus materiais de divulgação que suas soluções são focadas nos cidadãos (Cardullo \& Kitchin, 2019). A prefeitura de Dublin, na Irlanda, nomeou sua iniciativa baseada na Cidade Inteligente como Aberta, Engajada, Conectada, para indicar o envolvimento dos cidadãos. E, no Brasil, foi criada, em 2013, a Rede Brasileira de Cidades Inteligentes e Humanas por participantes da Frente Nacional de Prefeitos (RBCIH, 2020). 
Essa modificação no posicionamento de empresas e cidades consiste no estabelecimento de tentativas para conceber e implementar iniciativas de Cidades Inteligentes mais centradas no cidadão, a exemplo da realização de Living Labs e Hackatons. 0 primeiro caso compõe uma série de iniciativas trabalhadas com uma comunidade interessada e, usualmente, são lideradas por uma universidade, prefeitura ou empresa. No segundo, os cidadãos são convidados a participar de um evento, durante o fim de semana, para produzir protótipos de soluções técnicas para os problemas da cidade (Cardullo \& Kitchin, 2019).

No entanto, as ações muitas vezes não são formuladas de modo a integrar a participação dos cidadãos aos novos projetos no espaço urbano. Em razão de as atividades envolverem conhecimento computacional, geralmente, a atuação é mais instrumental do que o início de um debate político ou de uma situação que permita que os cidadãos façam reivindicações de direitos. Como explicam Cardullo \& Kitchin (2019, p. 5), "houve pouca tentativa para desempacotar sistematicamente as diversas maneiras pelas quais a participação do cidadão está sendo concebida e organizada na Cidade Inteligente"2.

Por essa razão, a partir do artigo pioneiro de Sherry Arnstein (1969), nomeado "Uma escada da participação cidadã" sobre os diferentes níveis de participação cívica nas decisões das cidades, Cardullo \& Kitchin (2019) propõem $O$ esqueleto para a participação do Cidadão Inteligente: uma ferramenta conceitual para indicar as diversas maneiras pelas quais a Cidade Inteligente enquadra os cidadãos. Cardullo \& Kitchin (2019) procuraram medir a inclusão, a participação e o empoderamento dos cidadãos em uma Cidade Inteligente, por meio de quatro formas e níveis de participação; são eles: Não Participação, Consumismo, Tokenismo ${ }^{3}$ e Poder do Cidadão. Tais formas de participação se dão conforme explicitamos a seguir:

1. Não Participação é sustentada por um forte impulso tecnocrático, em que os aspectos da cidade são tratados como problemas técnicos que também podem ser abordados por soluções técnicas. Os cidadãos são abordados em processos de coleta de dados e são considerados usuários com direitos e possibilidades limitados para alterar os resultados. Dessa maneira, a não participação se confunde com escolha, enquanto o usuário se torna consumidor.

2. Consumismo, como na Não Participação, é apoiado por uma forte estrutura tecnocrática. Nesse nível de participação, o espaço criado é de propriedade e administração particulares. Nesse enquadramento, os cidadãos/residentes se comportam como consumidores em um mercado de produtos e estilos de vida inteligentes, respondendo sobre projetos em fase de produção.

3. Tokenismo, em suas práticas mais amadurecidas, consiste em consulta e posicionamento, em que os cidadãos ganham uma voz, mas não possuem autonomia para liderar os projetos. Portanto, os cidadãos recebem informações sobre o desenvolvimento das ações propostas, são solicitados a colocar suas opiniões e podem sugerir alternativas e acréscimos às propostas.

4. Poder do Cidadão envolve uma redistribuição de poder dos gestores para os cidadãos, permitindo que as comunidades negociem e participem de trocas com lideranças estabelecidas para participar da administração do espaço urbano. Nessa perspectiva, o planejamento e a tomada de decisões são compartilhados, com regras e mecanismos básicos acordados para desenvolver projetos e resolver impasses.

Na aplicação em Cidades Inteligentes, segundo Cardullo \& Kitchin (2019), há poucos casos de Poder do Cidadão amplamente implementado. Onde a cocriação ocorre, geralmente ainda é por meio de Hackatons ou Living Labs. Em vista disso, artigos encontrados na literatura que abordam projetos de mobilidade, sociedade civil e, particularmente, a aplicação do Waze, em parceria com as prefeituras, não alcançaram discutir sobre a participação da população ou mesmo a adoção de uma metodologia que auxilie nas decisões sobre a utilização dos dados para projetos que beneficiem o espaço urbano.

No artigo Navigating Platform Urbanism, Ballon \& Graaf (2019) descrevem a participação das pessoas nas cidades por meio da intermediação das plataformas que coletam dados sobre a mobilidade. 0 trabalho apresenta a empresa Waze e o Programa Connected Citizens; entretanto, não discute como a parceria é

\footnotetext{
${ }^{2}$ Tradução da autora para: "There has been little attempt to systematically unpack conceptually the diverse ways citizen participation is being conceived and enacted in the smart city" (Cardullo \& Kitchin, 2019, p. 5).

3 Tokenismo advém do substantivo "token" que, em inglês, significa "símbolo". Essa expressão vem sendo empregada para fazer referência a esforços simbólicos de participação (Marchiori \& Sanchez, 2017).
} 
aplicada junto às prefeituras. Quanto ao tema da participação, restringe-se a analisar o controle dos dados fornecidos pelos usuários do Waze. E, no artigo In Waze We Trust: Algorithmic Governance of the Public Sphere, Graaf (2018) discute a aplicação dos dados coletados pelo Waze com mediação do governo eletrônico (e-gov) e suas práticas de serviços públicos digitais. Todavia, não investiga de que maneira a parceria entre o Waze e uma prefeitura se deu, para atender às demandas mais próximas das necessidades da mobilidade urbana.

No artigo "Em dados da multidão: análise da parceria entre Waze e prefeitura do Rio De Janeiro", Vicente (2017) aborda, a partir do contexto brasileiro, o primeiro acordo formal entre o Programa Connected Citizens do Waze e o setor público, para fornecimento de dados sobre mobilidade para o Centro de Operações do Rio de Janeiro. 0 autor discute a participação por meio da utilização das informações para discutir a implementação de ferramentas de transparência como parte da parceria. No entanto, não menciona caminhos para a prática da participação ou procedimentos para os dados serem aplicados, de maneira a responder aos desafios da mobilidade do Rio de Janeiro.

E no trabalho "Waze e centros de comando e controle urbanos: estudos de casos no Brasil", Pinto (2017) investiga a utilização dos dados coletados pelo Waze nos Centros de Operação do Rio de Janeiro, Petrópolis (RJ), Vitória (ES) e Juiz de Fora (MG), como também pela prefeitura de Salvador (BA). 0 estudo aborda situações pontuais, nas quais os dados sobre mobilidade foram utilizados, porém a participação da população ainda se encontra ou como provedora das informações ou apenas como recebedora, e não como parte atuante nas decisões sobre o planejamento de mobilidade.

Nessa perspectiva, este estudo propõe-se a investigar como foi realizada a parceria entre o programa Waze for Cities Data e a prefeitura de Joinville, tanto por meio da aplicação da metodologia Smart Mobility, quanto pelo modo como a população foi incentivada a participar, mediante a implementação de técnicas que permitiram que ela exprimisse as dificuldades e os objetivos para a mobilidade da cidade catarinense.

\section{A empresa Waze e o programa Waze for Cities Data}

Waze é uma empresa desenvolvedora de um aplicativo baseado em navegação GPS (Global Positioning System) com base na interação em comunidade dos usuários de dispositivos móveis (Vicente, 2017). Em 2019, registrou 115 milhões de usuários ativos por mês, presentes em 185 países. 0 Brasil é o quinto maior mercado da plataforma com 4 milhões de usuários mensais (Cilo, 2019).

O Waze começou a ser desenvolvido em 2006 em Israel, quando o engenheiro de software Ehud Shabtai percebeu que, para adicionar informações em um aparelho de navegação, precisaria de um mapa digital que pudesse ser editado e expandido. Shabtai também considerou que a plataforma deveria ser usada pela comunidade, que deveria ter acesso a ela para mapear as áreas. Assim, foi criada a primeira versão do aplicativo, que se chamava Freemap Israel (Levine, 2013).

São duas características que tornam o serviço do Waze diferente do de outros aplicativos que trabalham com informações de mapas. Primeiro, ele é construído graças à colaboração dos usuários e, segundo, ele se comporta como um mapa ao vivo, afirma Levine (2013). Todos os dias os mapas disponíveis no Waze sofrem alterações, de acordo com as informações adicionadas pelos motoristas; por isso, o diferencial do Waze vai além da navegação, uma vez que está mais focado na interação que o serviço promove em relação ao usuário (Levine, 2013).

Qualquer local que tenha interesse na utilização do Waze pode implantá-lo. No entanto, sua plataforma requer usuários iniciais que gerem e atualizem os mapas para torná-lo funcional. Para que os usuários do Waze interajam, eles precisam ter o aplicativo instalado em um smartphone ou tablet, e o aplicativo utiliza 0.23 MB de dados por hora (Waze, 2019). Dentre os 185 países em que o Waze está presente, apenas 13 possuem um mapa de base completo (Graaf, 2018). A estrutura de rede é importante para a qualidade das informações da plataforma. Quanto mais participantes, mais dados são coletados e, assim, há uma maior precisão no retorno das informações. 
Apesar de a interação ser uma ação importante para a eficiência do Waze, a empresa mantém o seu software fechado, como também detém as patentes. Ela compartilha apenas algumas das informações produzidas a partir da interação dos usuários. Com base em algoritmos de propriedade privada, os dados são filtrados e as informações são disponibilizadas por interfaces gráficas (Vicente, 2017).

Em 2012, o Waze começou a expandir seus serviços para além do aplicativo. 0 Connected Citizens Program foi criado em 2014, e, em 2018, ele se tornou o Waze for Cities Data. O projeto tem como objetivo a troca de dados sobre o trânsito com as cidades e com as concessionárias de serviço público (Waze, 2019).

O Brasil tem responsabilidade na geração do Connected Citizens Program, pois, em 2013, o Waze se tornou o fornecedor de dados de trânsito para o Centro de Operações do Rio de Janeiro (COR). Os APIs ${ }^{4}$ do Waze, integrados ao centro de controle do COR, encurtaram o caminho entre as informações sobre o trânsito e os alertas enviados pelos motoristas e os serviços de emergência, como também para os cidadãos do Rio de Janeiro (Connected Citizens Program, 2014).

O Connected Citizens Program iniciou em 2014 com dez locais parceiros: Rio de Janeiro, no Brasil; Barcelona, na Espanha; Jacarta, na Indonésia; Tel Aviv, em Israel; San Jose, na Costa Rica e; nos Estados Unidos, as cidades de Boston e Los Angeles, os estados de Utah e Flórida e, também, o Departamento de Polícia de Nova York (Wazeopedia, 2019). Em 2019, o Waze for Cities Data possuía mais de mil parceiros e, no Brasil, as cidades que participam do programa são: Juiz de Fora (MG), Petrópolis (RJ), Rio de Janeiro (RJ), São Paulo (SP), Vitória (ES) e Joinville (SC).

\section{Procedimentos metodológicos}

A presente pesquisa consiste em uma análise exploratória de um estudo de caso, de caráter qualitativo, com o objetivo de examinar a metodologia do projeto Smart Mobility, desenvolvida e aplicada pela Secretaria de Planejamento Urbano e Desenvolvimento Sustentável (Sepud) de Joinville (SC), para a implementação do programa Waze for Cities Data, com base nos dados coletados pelo Waze sobre o tráfego no seu perímetro urbano. Para tal, o instrumento de coleta de dados se deu por meio de uma observação sistemática/não participante.

Nesse tipo de observação, o pesquisador não interage com o objeto de estudo. Na coleta de dados, ele já sabe quais informações são significativas para tal. Portanto, antes de executar a observação sistemática, é realizado um plano de execução (Gerhardt et al., 2009).

Para este estudo, o plano de execução iniciou-se com a pesquisa do referencial bibliográfico sobre o conceito de Cidades Inteligentes, Big Data aplicado no planejamento urbano orientado a dados e participação das pessoas para projetos mais centrados no cidadão. Na sequência, analisou-se o histórico da empresa Waze, que, através do seu aplicativo, coleta dados sobre o tráfego nas cidades e informa os motoristas que utilizam seu serviço. Posteriormente, foi realizada uma visita de observação à cidade de Joinville.

Dessa maneira, o roteiro para a coleta de dados apresentou as seguintes etapas: primeiro, foi contatado o gestor da Sepud para o agendamento da visita de observação. A visita ocorreu em setembro de 2019 na sede da Secretaria. Essa observação sistemática, que teve como participantes os profissionais da área da mobilidade e gestão da Sepud, partiu das seguintes questões:

1. Qual era a demanda da cidade de Joinville para utilizar os dados do Waze?

2. Como foram aplicados os dados coletados pelo Waze?

3. Quais os próximos desdobramentos a partir da aplicação do projeto?

Dessa maneira, essas questões direcionaram a observação e também a coleta de dados para esta pesquisa. Com base nas informações encontradas na visita a Joinville e também no material disponibilizado sobre a aplicação do projeto Smart Mobility, foi possível, então, desenvolver este estudo.

\footnotetext{
${ }^{4}$ API é a sigla para Application Programming Interface: é uma interface que permite o acesso, via programação, às funcionalidades de um sistema e permite a troca de informações entre dois ou mais sistemas (Ciriaco, 2009).
} 


\section{Resultados e discussão}

\section{Joinville e a aplicação do Waze for Cities Data}

Joinville possui uma população de 590.466 habitantes (IBGE, 2019) e uma frota de 410 mil veículos (Prefeitura de Joinville, 2019). Está localizada na região norte do estado de Santa Catarina; considerado de médio porte, é o terceiro maior município da região Sul do Brasil, depois de Porto Alegre e Curitiba.

Com o objetivo de implementar uma gestão mais direcionada aos dados, o primeiro movimento realizado pela Secretaria de Planejamento Urbano e Desenvolvimento Sustentável (Sepud) foi organizar a grande quantidade de dados internos que se encontravam espalhados pelas secretarias e extrair valor deles. Por consequência, foi evidenciada a necessidade de mais dados externos, especificamente os que retratassem a situação da mobilidade do município em uma frequência mais próxima à realidade. Essa situação exemplifica, dentro do movimento da gestão das cidades, a importância dos dados para apoiar os processos de tomada de decisão e planejamento (Batty, 2013).

A partir desse foco, após a Sepud conhecer a parceria entre o Waze e o Centro de Operações do Rio de Janeiro, percebeu-se que também era possível aplicar o programa Waze for Cities Data em Joinville. Em 2017, tendo como impulsionadora a Lei n. 12.587/12, conhecida como Lei da Mobilidade Urbana, a qual determina aos municípios a tarefa de planejar e executar a política de mobilidade urbana, como também a ampla utilização dos dados para benefício do município, a Prefeitura de Joinville começou a organizar o projeto Smart Mobility.

A mobilidade é uma das infraestruturas mais importantes e desafiadoras que tem como objetivo apoiar o funcionamento da área urbana. Como explica Benevolo et al. (2016), há seis principais objetivos que a Mobilidade Inteligente busca cumprir; quais sejam: 1. redução da poluição; 2. redução do congestionamento; 3. aumento da segurança das pessoas; 4. redução da poluição sonora; 5 . melhoria da velocidade de deslocamento e; 6. redução de custos de viagem.

Desse modo, objetivando-se uma Mobilidade Inteligente, além da parceria com o Waze, o projeto Smart Mobility é apoiado por instituições e ferramentas que contribuem com as informações sobre a mobilidade em Joinville, como indica a Tabela 1.

Tabela 1 - Principais atores e suas contribuições no projeto Smart Mobility

\begin{tabular}{|c|c|}
\hline Atores & Contribuições \\
\hline Empresas de tecnologia & $\begin{array}{l}\text { Exemplo: Empresa que implementou o sistema de radar do município que } \\
\text { opera em } 100 \text { pontos espalhados pela cidade e informa o fluxo de } \\
\text { veículos/hora a cada } 15 \text { minutos. }\end{array}$ \\
\hline $\begin{array}{l}\text { Ampla infraestrutura de hardware, software } \\
\text { e Internet das Coisas }\end{array}$ & $\begin{array}{l}\text { - armazenamento em nuvem e servidores em datacenter; } \\
\text { - rede de comunicação MESH com aproximadamente } 10 \text { mil pontos para } \\
\text { interligar os sensores de Internet das Coisas. }\end{array}$ \\
\hline Prefeitura de Joinville & Informações cadastrais e georreferenciadas das 2 mil vias do município. \\
\hline $\begin{array}{l}\text { Órgãos públicos cujo trabalho é ligado à } \\
\text { mobilidade, tais como: Samu; Detran; } \\
\text { Guarda Municipal; Hospital Municipal; Corpo } \\
\text { de Bombeiros Voluntários. }\end{array}$ & Informam sobre o local do acidente e veículos envolvidos. \\
\hline $\begin{array}{l}\text { Softwares de código aberto para a } \\
\text { simulação de tráfego. }\end{array}$ & $\begin{array}{l}\text { - Sumo: simulação de rotas; } \\
\text { - OpenStreetMap: plataforma de mapeamento colaborativo; } \\
\text { - QGIS: análise de dados georreferenciados. }\end{array}$ \\
\hline
\end{tabular}

Fonte: Elaborado pela autora (2020).

A empresa Waze se destaca no projeto Smart Mobility como o principal fornecedor de informações. Por meio do projeto Waze for Cities Data, a empresa entrega dados dos incidentes e acidentes reportados no aplicativo do Waze no perímetro urbano. Por exemplo: engarrafamentos, tamanho da fila de congestionamento em metros, velocidade dos veículos em fila em quilômetro/hora e tempo de atraso gerado pela fila medido em segundos. Dessa maneira, há mais informações direcionadas às operações de 
gestão viária e focadas na redução dos congestionamentos. Essa utilização dos dados, no planejamento de Joinville, coaduna-se com algumas considerações de Batty (2013), quando o autor afirma que

[o] Big Data está certamente enriquecendo nossas experiências de como as cidades funcionam, e está oferecendo muitas novas oportunidades de interação social e tomadas de decisão mais informadas com relação ao nosso conhecimento sobre a melhor forma de interagir nas cidades ${ }^{5}$ (Batty, 2013, p. 277).

Assim sendo, o projeto Smart Mobility, além de realizar a parceria entre instituições que coletam dados sobre a mobilidade na cidade de Joinville, também se preocupou em desenvolver uma metodologia que aprimorasse a compreensão dos dados coletados. Todas as etapas e ações tornam as decisões para mobilidade em Joinville mais voltadas para o benefício dos cidadãos.

A partir dos dados fornecidos pelo Waze, a metodologia criada pela Sepud é aplicada em cinco etapas, como mostra a Figura 1, e elas são denominadas: 1) Diagnóstico e priorização; 2) Estimativa de demanda; 3) Simulação; 4) Análise de cenários; e 5) Medição de resultados. E o principal objetivo de cada etapa é: 1) Quais ruas deverão sofrer intervenção primeiro?; 2) Qual o volume de tráfego nessas vias?; 3) Como o trânsito vai responder a possíveis intervenções?; 4) Qual a melhor proposta de intervenção?; 5) Como o trânsito realmente respondeu à intervenção.

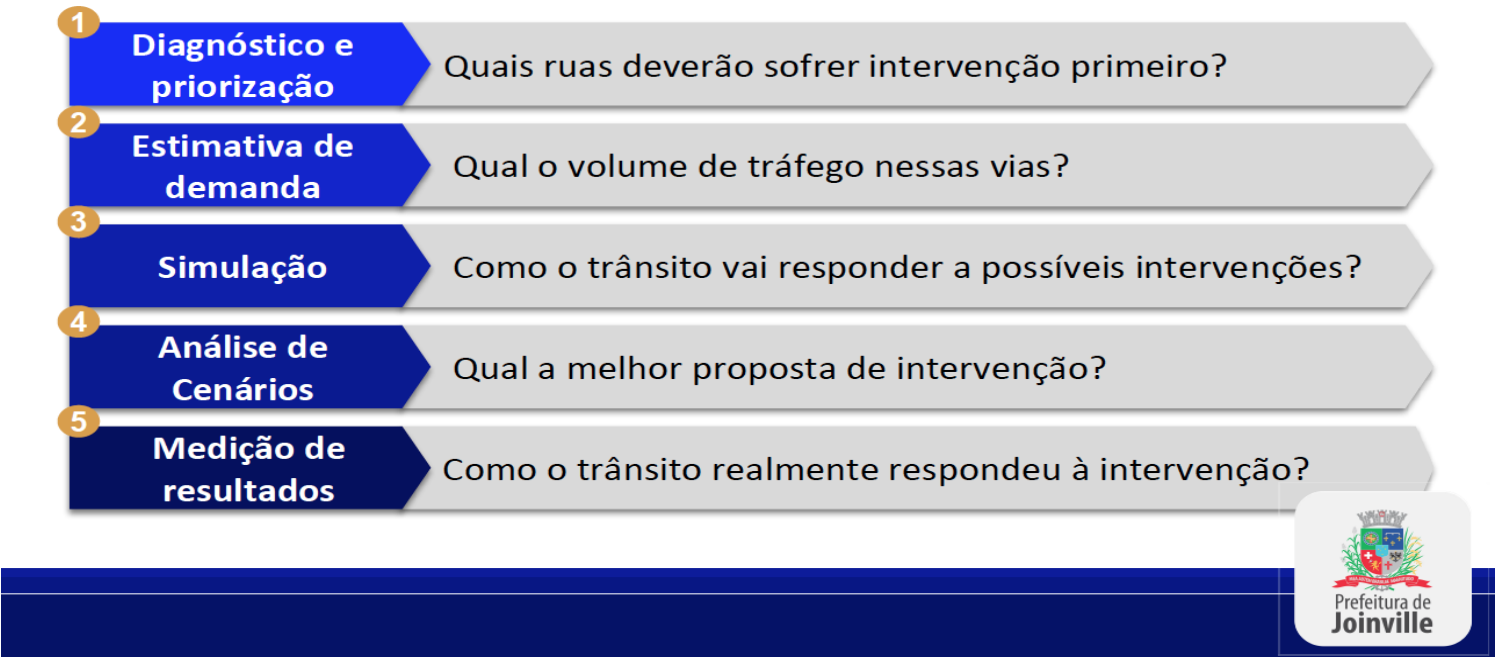

Figura 1 - Cinco etapas da metodologia do projeto Smart Mobility. Fonte: Prefeitura de Joinville (2019).

Os dados são recebidos, via Waze, por meio de um log que a empresa disponibiliza para a Sepud; e a atualização é feita a cada 15 minutos. Em Joinville, há 2 mil ruas que são divididas em 19 mil trechos, dos quais cada um é aproximadamente o tamanho de uma quadra. Com o objetivo de decidir qual seria a primeira rua a ter uma intervenção realizada pelo projeto Smart Mobility, foi criado pela Sepud o cálculo para se avaliar o valor da criticidade.

Para se chegar a esse valor, primeiro são recebidos do Waze o comprimento de fila, o atraso médio e a velocidade média. Então, a probabilidade de congestionamento é calculada pela Sepud, que considera a frequência com que uma determinada rua registra o congestionamento em relação a todas as coletas de dados realizadas durante o dia.

Na sequência, o valor da criticidade é feito para cada trecho da via a partir da multiplicação entre a probabilidade de congestionamento e o atraso médio. Como há algumas ruas que cortam toda a cidade, $o$ cálculo por trecho é mais próximo à situação da mobilidade (Prefeitura de Joinville, 2019).

Sobre a participação dos cidadãos, a necessidade de uma comunicação mais assertiva entre a prefeitura e os moradores foi manifestada em razão da mudança da gestão da mobilidade. Diferentemente das

\footnotetext{
5 Tradução da autora para: "Big data is certainly enriching our experiences of how cities function, and it is offering many new opportunities for social interaction and more informed decision-making with respect to our knowledge of how best to interact in cities" (Batty, 2013, p. 277).
} 
audiências que não resultavam em medidas produtivas, foi criada, pela Sepud, uma metodologia de consulta pública. 0 método de participação se dá por meio da realização de três reuniões com a comunidade do bairro a ter a via alterada, ocasiões nas quais são discutidas as três etapas do projeto Smart Mobility.

Na primeira reunião, um diagnóstico da mobilidade da região é exposto e a população é consultada sobre os anseios e desafios no deslocamento pelo bairro. No segundo encontro, a Sepud apresenta uma proposta de intervenção de acordo com os dados, a simulação de tráfego e os interesses dos moradores que foram apresentados na reunião anterior. Na terceira etapa, o projeto final é apresentado, a partir das possíveis alterações discutidas durante a segunda reunião e, o cronograma de implantação também é anunciado (Prefeitura de Joinville, 2019).

Para que as reuniões sejam mais dinâmicas e objetivas, no momento do encontro, a comunidade é dividida em grupos, os quais recebem folhas de sulfite e blocos de papel para anotar suas ideias. Ao final, os grupos apresentam o que foi debatido e as propostas que dali surgiram. Após a reunião, o material resultante é, então, tabulado pela Secretaria. Desde sua criação, a metodologia de participação já foi aplicada em cinco pontos da cidade e os intervalos entre as reuniões variam de acordo com o tempo de execução de cada projeto (Prefeitura de Joinville, 2019).

De acordo com "O esqueleto para a participação do Cidadão Inteligente", proposto por Cardullo \& Kitchin (2019), observa-se que na participação dos cidadãos no projeto Smart Mobility, o nível exercido é o Tokenismo. Ou seja, os cidadãos são consultados, suas opiniões registradas, no entanto, ainda não possuem autonomia para o exercício da ação.

\section{Aplicação do projeto Smart Mobility na Rua Ottokar Doerffel}

Como primeiro estudo de caso, essa metodologia foi aplicada na Rua Ottokar Doerffel, principal acesso à cidade de Joinville. A Figura 2 ilustra o perímetro urbano que teve a sua área modificada devido a uma alteração: a linha tracejada em azul indica a Rua Ottokar Doerffel, o círculo em vermelho o local da rua onde foi implementada a rotatória, e a via destacada em rosa é a Rodovia Federal BR-101, conhecida também como Rodovia Governador Mário Covas, que permite a ligação de Joinville a outras cidades.

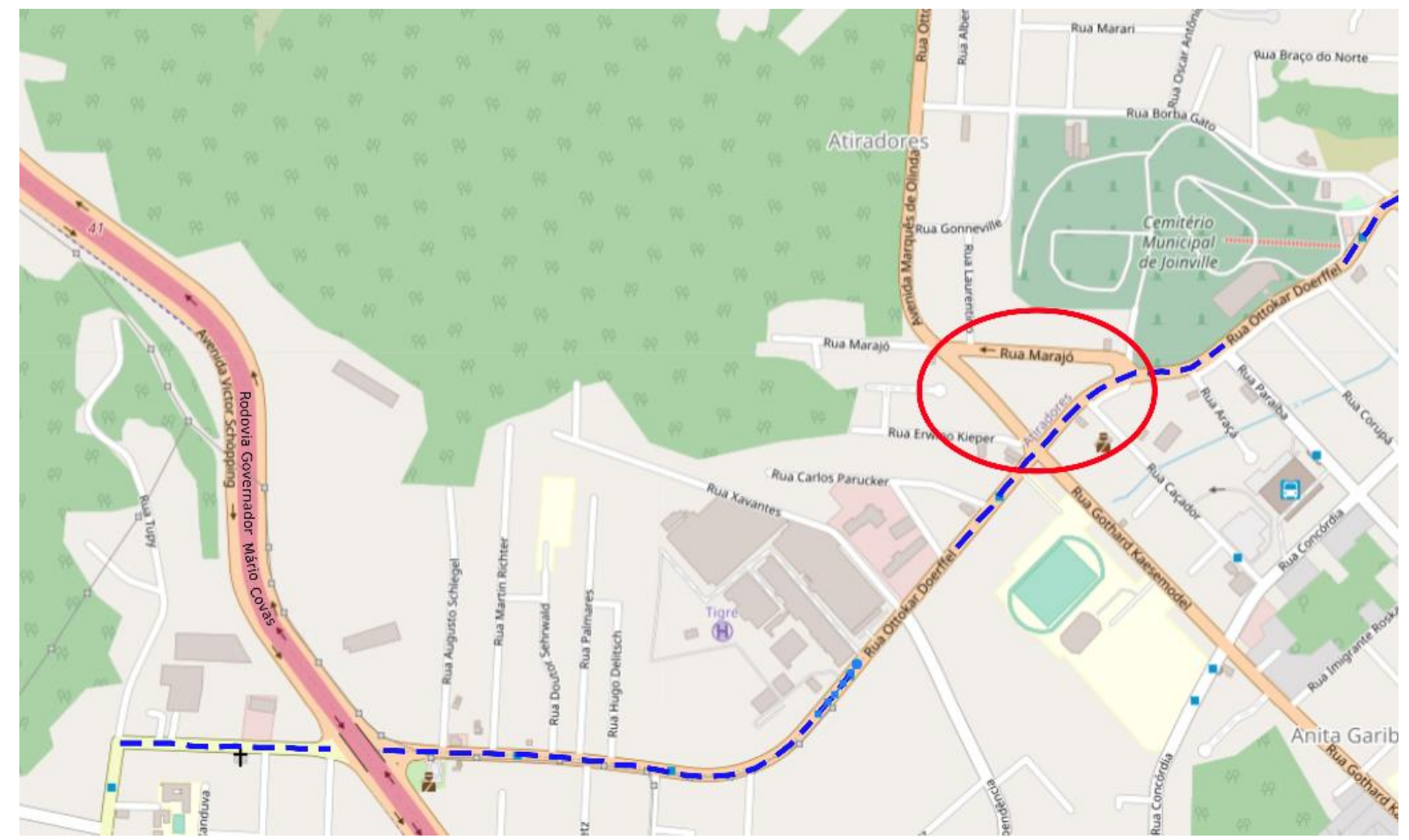

Figura 2 - Perímetro urbano de Joinville com a indicação de onde foi realizada a alteração na Rua Ottokar Doerffel. Fonte: OpenStreetMap (2020). 
A partir da etapa 1, Diagnóstico e priorização, como apresenta a Tabela 2, foi calculado, pela Sepud, o valor final da criticidade por logradouro, isto é, foi verificada a média das criticidades de todos os trechos que compõem a rua. Dessa maneira, esse parâmetro indica os locais onde há maior probabilidade de congestionamento no horário de pico e onde esse fenômeno provoca um grande atraso para a população (Prefeitura de Joinville, 2019).

Com base nesse cálculo da criticidade, as ruas foram organizadas em um ranking, segundo o qual a Rua Ottokar Doerffel foi classificada com 751,0. Isso a qualificou como a segunda via mais congestionada do município. Também, por ser a via que registrava inúmeros acidentes anualmente, ela foi escolhida como o primeiro trecho no qual seria aplicada a metodologia do projeto Smart Mobility.

Tabela 2 - Ranking de criticidade das ruas de Joinville realizado na etapa 1 da metodologia do projeto Smart Mobility

\begin{tabular}{lll}
\hline & Rua & Criticidade \\
\hline 01 & Dona Francisca & 934,4 \\
02 & Ottokar Doerffel & 751,0 \\
03 & Guanabara & 720,8 \\
04 & Quinze de Novembro & 692,9 \\
05 & Prefeito Helmuth Fallgatter & 574,6 \\
\hline
\end{tabular}

Fonte: Prefeitura de Joinville (2019).

Após a escolha do local onde seria implementado o projeto, o trecho entre as ruas Ottokar Doerffel e Marquês de Olinda passou por três etapas - como mostra a Figura 3 - para que fossem elaboradas as propostas de execução na área. Primeiro, a região foi fotografada por drones. Na sequência, no software Sumo, a região foi calibrada até aproximar a simulação da situação real na área. Desse modo, a partir do cenário das vias desenvolvido com a ajuda do software, diversas propostas foram elaboradas até a definição daquela capaz de reduzir o congestionamento da via.
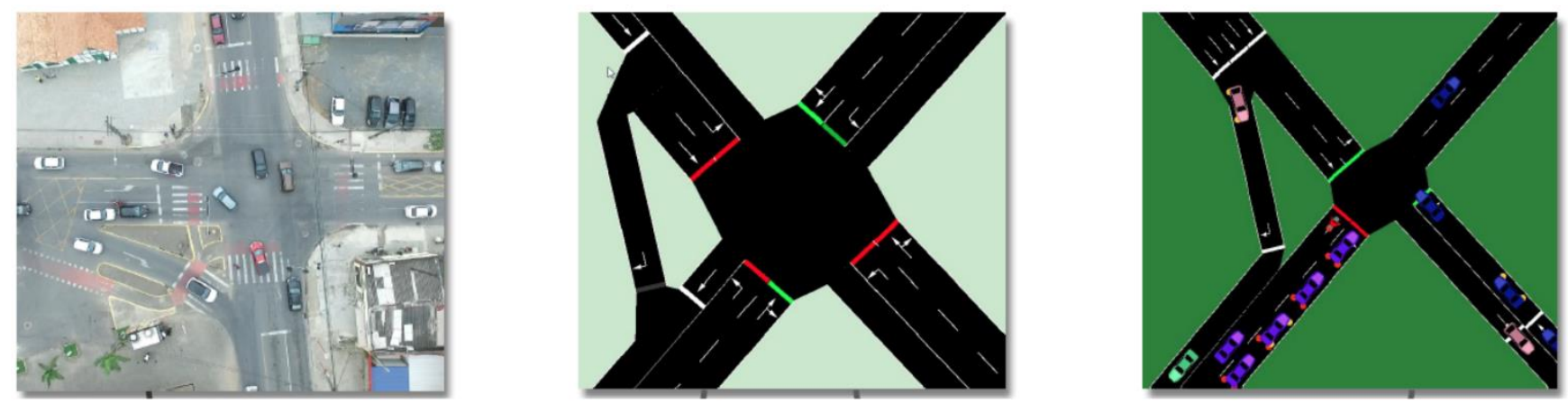

Figura 3 - Ełapa 3: simulação e calibração no software Sumo do cenário encontrado na Rua Ottokar Doerffel. Fonte: Prefeitura de Joinville (2019).

Na sequência, a etapa realizada foi a 4, Análise de cenários. A partir dos resultados obtidos, oito cenários foram desenvolvidos. Dentre eles, três foram considerados para a implementação na via, como mostra a Tabela 3. No cenário sem alterações, o tempo médio de viagem era de 11,6 minutos, o tempo médio perdido de 9,8 minutos e o tempo máximo de viagem de 38,6 minutos.

Com base nas informações encontradas na etapa 3, Simulação, três principais cenários se destacaram para diminuir o congestionamento na Rua Ottokar Doerffel, como apresenta a Tabela 3. Apesar do cenário 3 ter apresentado os menores resultados, ele indicava a retirada de um semáforo, o que poderia tornar o deslocamento do pedestre mais inseguro. Por isso, o cenário 2 foi o escolhido por ser o mais favorável a ser 
implementado. Em relação ao cenário que havia na Rua Ottokar Doerffel, a partir dos dados coletados, ele apresentou uma melhoria de 6,2 minutos para o tempo médio de viagem, 6,4 minutos para o tempo médio perdido e 17,7 minutos para o tempo máximo de viagem.

Tabela 3 - Ełapa 4 - análise de cenários com escolha do cenário 2 para alteração da Rua Ottokar Doerffel.

\begin{tabular}{lccccc}
\hline & Cenário sem alterações & Cenário 1 & Cenário 2 & Cenário 3 & Melhoria \\
\hline Tempo Médio de Viagem (min) & 11,6 & 8,4 & 5,4 & 3,5 & 6,2 \\
Tempo Médio Perdido (min) & 9,8 & 6,6 & 3,4 & 1,5 & 6,4 \\
Tempo Máximo de Viagem (min) & 38,6 & 23,7 & 20,9 & 8,8 & 17,7 \\
\hline
\end{tabular}

Fonte: Prefeitura de Joinville (2019).

A execução do cenário 2 consistiu na implementação de uma rotatória de uma quadra inteira e de baixo custo, como mostra a Figura 4. A alteração do tráfego, para esse cenário, beneficiou não apenas os motoristas, mas também os pedestres e a economia do município.

Os pedestres foram beneficiados com uma travessia mais segura na frente da fábrica da Tigre. Os motoristas diminuíram, em média, 6,2 minutos no seu deslocamento, o que resultou, para os cidadãos, em um ganho de tempo de aproximadamente 3 dias e 7 horas por ano, período que não precisam desperdiçar no trânsito e que podem utilizar em prol de sua produtividade e lazer.

Por consequência, o município também obteve um retorno de $\mathrm{R} \$ 7.652 .251,72 \mathrm{em}$ produtividade/ano. Esse cálculo é o produto de três fatores: 1. a diminuição do tempo nos congestionamentos, 2. a quantidade de pessoas que trafegam nos horários de maior movimento, como no começo do período da manhã e final do período da tarde e 3. valor da hora/trabalho dos cidadãos de acordo com o salário médio de Joinville (Prefeitura de Joinville, 2019).

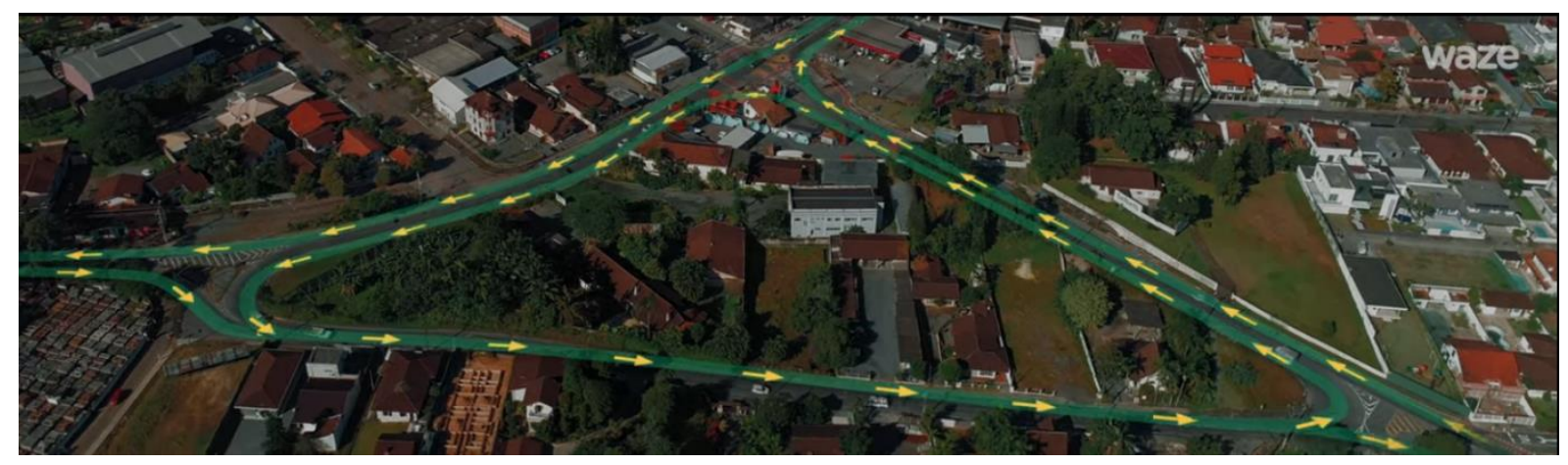

Figura 4 - Implementação da obra na rotatória que contempla as ruas Ottokar Doerffel, Marajó e Marquês de Olinda. Fonte: Waze (2019).

Depois da implementação da obra, na etapa 5, Medição de resultados, a Sepud constatou uma alteração na velocidade média do tráfego em diferentes pontos da Rua Ottokar Doerffel. A partir das informações disponibilizadas pelo Waze minuto a minuto - comprimento de fila, atraso médio e velocidade média verificou-se que toda a extensão da Rua Ottokar Doerffel recebeu benefícios significativos quanto à diminuição do congestionamento, tanto no período da manhã, quanto no período da tarde, como apresenta a Tabela 4 .

Observa-se que, no período da manhã (Tabela 4), a exemplo do ponto da Rua Ottokar Doerffel no sentido Centro, antes da intervenção era registrada uma velocidade média de $23,4 \mathrm{~km} / \mathrm{h}$ e depois da intervenção houve um aumento para $26,5 \mathrm{~km} / \mathrm{h}$ (Waze, 2019).

E no mesmo ponto, no período da tarde, como mostra a Tabela 4, antes da intervenção, a velocidade média registrada era de $23,7 \mathrm{~km} / \mathrm{h}$; depois da alteração para a rotatória, esse valor aumentou para 26,6 $\mathrm{km} / \mathrm{h}$. Uma das áreas com significativa alteração da velocidade média foi na Rua Ottokar Doerffel, na entrada da Rua Marajó no sentido Rodovia Federal BR-101. Antes era registrada a velocidade média de $21 \mathrm{~km} / \mathrm{h}, \mathrm{e}$, após a intervenção, passou a ser medido o máximo de $30 \mathrm{~km} / \mathrm{h}$ que é permitido na via. 
Tabela 4 - Velocidades médias (km/h) antes e depois da intervenção na Rua Ottokar Doerffel

\begin{tabular}{|c|c|c|c|c|}
\hline \multirow{2}{*}{ Local } & \multicolumn{2}{|c|}{ Manhã } & \multicolumn{2}{|c|}{ Tarde } \\
\hline & Antes & Depois & Antes & Depois \\
\hline Rua Ottokar Doerffel sentido Rodovia Federal BR-101 & 28.8 & 30 & 22.6 & 29.7 \\
\hline Rua Ottokar Doerffel sentido Centro & 23.4 & 26.5 & 23.7 & 26.6 \\
\hline Rua Marquês de Olinda sentido Rua Ottokar Doerffel & 29.7 & 29.8 & 23.2 & 26.8 \\
\hline $\begin{array}{l}\text { Rua Ottokar Doerffel na entrada da Rua Marajó sentido } \\
\text { Rodovia Federal BR-101 }\end{array}$ & 29.1 & 30 & 21 & 30 \\
\hline Rua Ottokar Doerffel na entrada da Rua Marajó sentido Centro & 29.8 & 30 & 29.3 & 30 \\
\hline
\end{tabular}

Fonte: Prefeitura de Joinville, 2019.

Esse planejamento da cidade de Joinville, focado na utilização dos dados e aplicado através do projeto Smart Mobility, recebeu seu reconhecimento nacional e foi ganhador do prêmio InovaCidade de 2019, promovido pelo Instituto Smart City Business America (Schiavo, 2019). Também se tornou um exemplo internacional da parceria com o Waze for Cities Data e é divulgado pela empresa como uma referência para outras cidades no mundo.

A secretaria ainda propõe três principais ações para transpor a experiência do projeto em outras cidades: a realização de Hackatons sobre mobilidade, a colaboração entre os municípios que estejam trabalhando com a aplicação do Smart Mobility e uma articulação denominada hélice tripla. Tal articulação é composta pela prefeitura, empresas e universidades. A primeira contribui com os dados e recebe a aplicação de ações com inteligência na mobilidade urbana; a segunda fornece capital e tem como benefício a participação em um ecossistema estabelecido de inovação, como também visibilidade em um projeto para os cidadãos; a terceira participa com profissionais qualificados. 0 projeto, por sua vez, possibilita a realização de publicações que possam incentivar outros pesquisadores e municípios (Prefeitura de Joinville, 2019).

Dessa maneira, a Sepud se coloca à disposição de outras prefeituras que necessitem de auxílio na implementação e, também, para visitas com o objetivo de conhecer o cotidiano da aplicação em Joinville. Assim como disponibiliza na plataforma GitHub, a parte técnica do projeto, como a metodologia e os códigos utilizados na programação.

Nesse sentido, para que um município possa dar início à aplicação dos dados coletados em seu perímetro urbano, para melhoria em suas vias, além das etapas executadas pelo projeto Smart Mobility, ele deve implementar as ações listadas na Tabela 5, cada qual mencionada neste estudo.

Tabela 5 - Ełapas para implementação dos dados coletados pelo Waze para melhoria da mobilidade no município

\begin{tabular}{|c|c|}
\hline 1) & Demanda do espaço urbano. \\
\hline 2) & Possibilidade de utilização dos dados do Waze na cidade. \\
\hline 3) & Contato com o Waze. \\
\hline 4) & Parceria com o programa Waze for Cities Data. \\
\hline 5) & Aplicação de uma metodologia de implementação, por exemplo, a do projeto Smart Mobility. \\
\hline 6) & Consulta com a população. \\
\hline 7) & Implementação de alteração na via. \\
\hline 8) & Nova coleta de dados sobre a mobilidade através do Waze. \\
\hline 9) & Verificação dos resultados. \\
\hline
\end{tabular}


O projeto Smart Mobility e sua aplicação na cidade de Joinville demonstram como a utilização de dados resultou em ações importantes para o espaço urbano, a partir da participação de diversos setores. Os dados coletados com o auxílio dos motoristas foram transmitidos pelo Waze para o poder público e, dessa maneira, os gestores puderam, por meio de uma metodologia, tomar uma decisão e disponibilizar uma solução para a população, que pôde participar da implementação por meio do nível tokenismo.

Também, a análise do estudo de caso revelou que essa forma de gestão orientada aos dados pode levar a uma administração mais comprometida com os fatos e com a aplicação de soluções para a população. Assim, as práticas governamentais implementadas alinham-se mais à realidade vivenciada pelos cidadãos do que aos interesses políticos.

\section{Considerações finais}

O caso de Joinville demonstra que o aplicativo Waze delineou um caminho para a aplicação dos dados coletados no espaço urbano e oferece uma metodologia para sua implementação. No entanto, a Sepud verificou que esse método é eficiente apenas para regiões pequenas. Para áreas com maior abrangência, essa aplicação se torna inviável, pois dependeria de uma grande quantidade de pontos de contagem de tráfego, o que, consequentemente, aumentaria o tempo para se chegar ao resultado do estudo e, em seguida, sua aplicação (Prefeitura de Joinville, 2019).

Por essa razão, e devido à sequência do desenvolvimento do projeto Smart Mobility, a Sepud está buscando desenvolver um modelo de simulação que seja independente das contagens de campo. 0 novo modelo também contará com a participação dos dados do Waze, porém, utilizará os dados coletados a partir de 95 controladores de velocidade que gerarão uma matriz Origem Destino (OD) através de um modelo matemático denominado Modelo Gravitacional, utilizado na Engenharia de Tráfego. Após o cálculo da matriz, ela será inserida em um simulador de tráfego e calibrada através dos dados do Waze. Dessa maneira, há a possibilidade para monitorar propostas de médio e grande portes, o que permite uma cobertura completa do tráfego de Joinville no simulador.

Outro desafio para uma gestão urbana baseada em dados, que foi encontrado por este estudo e apontado pela prefeitura de Joinville, é a falta da continuidade dos projetos pelos próximos governos. No Brasil, as disputas entre os partidos políticos dificultam a consolidação de projetos que relacionam tecnologia, infraestrutura e políticas públicas, ou seja, ações que necessitam de mais de quatro anos para serem elaboradas, testadas, executadas e, enfim, se tornarem uma rotina na cidade.

Por outro lado, apesar dos desafios políticos existentes, a parceria entre o programa Waze for Cities Data e a Prefeitura de Joinville demonstra que a discussão e a execução de projetos relacionadas a Cidades Inteligentes se intensificam no Brasil e que as cidades de pequeno e médio portes brasileiras se estabelecem como plataformas de verificação para projetos de tecnologia que beneficiem os municípios, inclusive as metrópoles.

Este estudo também observou a intensificação do uso dos dados coletados por empresas na gestão das cidades para encontrar soluções mais próximas à realidade da população. Além disso, apresentou a possibilidade de projetos de Cidades Inteligentes mais centrados no cidadão, nos quais ele pode desempenhar ações na concepção, desenvolvimento e governança. Para futuras análises, buscam-se outros projetos que contemplem a aplicação das informações geradas pelos dados produzidos nos espaços urbanos brasileiros e atuem como projetos de referência para outros países.

\section{Agradecimentos}

Agradeço à Sepud, ao secretário de Planejamento Urbano e Desenvolvimento Sustentável Danilo Conti e ao engenheiro Guilherme Belegante, pelas informações concedidas sobre a aplicação do projeto Smart Mobility. 


\section{Referências}

Alvarez, R. (2017). The Relevance of Informational Infrastructures in Future Cities. Field Actions Science Reports, 17, 12-15. Recuperado em 5 de dezembro de 2019 de http://journals.openedition.org/factsreports/4389

Arnstein, S. R. (1969). A ladder of citizen participation. Journal of the American Institute of Planners, 35(4), $216-224$. https://doi.org/10.1080/01944366908977225

Ballon, P., \& Graaf, S. van der. (2019). Navigating Platform Urbanism. Technological Forecasting \& Social Change, 142, 364372. https://doi.org/10.1016/j.techfore.2018.07.027

Batty, M. (2013). Big data, smart cities and city planning. Dialogues in Human Geography, 3(3), 274-279. https://doi.org/10.1177/204382061351339

Batty, M. (2016). Big Data and The City. Built Environment, 42(3), 321-336. https://doi.org/10.2148/benv.42.3.321

Benevolo, C., Dameri, R. P., \& D’Auria, B. (2016). Smart mobility in Smart City: Action taxonomy, ICT intensity and public benefits. Empowering organizations. Enabling platforms and artefacts, 11, 13-28. https://doi.org/10.1007/978-3-31923784-8_2

Brasil (2012, 04 de janeiro). Lei n. 12.587/12, de 3 de janeiro de 2012. Institui as Diretrizes da Política Nacional de Mobilidade Urbana. Brasília: Diário Oficial da União, seção 1.

Buosi, M. de A. (2018). Mobilidade inteligente: proposta de framework de Big Data analytics para análise de dados de mobilidade urbana em uma Smart City (Dissertação de mestrado). Programa de Pós-graduação em Administração, Universidade Estadual de Santa Catarina, Florianópolis.

Cardullo, P., \& Kitchin, R. (2019). Being a 'citizen' in the smart city: up and down the scaffold of smart citizen participation in Dublin, Ireland. GeoJournal, 84, 1-13. https://doi.org/10.1007/s10708-018-9845-8

Cilo, N. (2019). Brasil é o $5^{\circ}$ maior mercado do Waze, diz gerente-geral do app no país. Brasília: Correio Brasiliense. Recuperado em 5 de dezembro de 2019, de https://www.correiobraziliense.com.br/app/noticia/economia/2019/03/18/internas_economia,743566/entrevista-comleandro-esposito-gerente-geral-do-waze-no-brasil.shtml

Ciriaco, D. (2009). O que é API? Tecmundo. Recuperado em 8 de dezembro de 2019, de https://www.tecmundo.com.br/programacao/1807-o-que-e-api-.htm

Connected Citizens Program. (2014). Waze Connected Citizens Overview Packet - with Technical Details. Recuperado em 8 de dezembro de 2019, de https://docs.google.com/document/d/1msH86f0Uh9DoRT0srC1a-MMYjhwF8hTqHiR4AeS72U/edit

Cunha, M. A. V. C. D., Henriques, A. C. V., \& Meirelles, F. d. S. (2020). Big data analytics: achievements, challenges, and research trends. Independent Journal of Management \& Production, 11(4), 1201-1222.

http://dx.doi.org/10.14807/ijmp.v11i4.1085

De Waal, M., \& Dignum, M. (2017). The citizen in the smart city. How the smart city could transform citizenship. Information Technology, 59(6), 263-273. https://doi.org/10.1515/itit-2017-0012

Firmino, R. J., \& Simão, M. de M. B. (2019). A construção social de um sistema de mobilidade inteligente: mapeando controvérsias no caso do Swipass. Cadernos Metrópoles, 21(44), 331-354. https://doi.org/10.1590/2236-9996.20194414Gerhardt, T. E., Ramos, I. C., Riquinho, D. L., \& Santos, D. L. dos. (2009). Estrutura do Projeto de Pesquisa. In T. E. Gerhardt, \& D. T. Silveira (Eds.), Métodos de Pesquisa (p. 65-88). Porto Alegre: Editora da UFRGS.

Graaf, S. van der. (2018). In Waze We Trust: Algorithmic Governance of the Public Sphere. Media and Communication, 6(4), 1-10. http://dx.doi.org/10.17645/mac.v6i4.1710

Harvey, D. (1996). Do gerenciamento ao empresariamento: a transformação da administração urbana no capitalismo tardio. In L. O. da Silva, \& M. C. da Silva Leme (Orgs.), Espaço \& Debates: Revista de Estudos Regionais e Urbanos (1a ed., Vol. 39, p. 48-64). São Paulo: Neru.

Instituto Brasileiro de Geografia e Estatística - IBGE. (2019). Joinville. Rio de Janeiro: IBGE. Recuperado em 10 de dezembro de 2019, de https://cidades.ibge.gov.br/brasil/sc/joinville/panoramaKitchin, R. (2014). The real-time city? Big data and smart urbanism. GeoJournal, 79, 1-14. http://dx.doi.org/10.2139/ssrn.2289141Kitchin R. (2016). The ethics of smart cities and urban science. Philosophical Transactions of the Royal Society A: Mathematical, Physical and Engineering Sciences, 374 (2083), 1-15. https://doi.org/10.1098/rsta.2016.0115 
Kitchin, R. (2017). Big Data. The International Encyclopedia of Geography, 1, 1-3. https://doi.org/10.1002/9781118786352.wbieg0145

Levine, U. (2013). Afinal, quais são os segredos de sucesso do Waze?. Recuperado em 5 de dezembro de 2019, de https://administradores.com.br/noticias/afinal-quais-sao-os-segredos-de-sucesso-do-waze

Marchiori, P.Z., \& Sanchez, C.S. (2017). Elementos que caracterizam a participação popular no contexto das iniciativas de governo aberto: revisão sistemática da literatura. In IV Encontro Brasileiro de Administração Pública (Vol. 1, p. 50-72). João Pessoa, Paraíba: SBAP.

Menezes, F. (2018). Urbanismo movido a dados: o Big Data como ferramenta de gestão urbana (Dissertação de mestrado). Programa de Pós-Graduação em Gestão Urbana da Escola de Arquitetura e Design, Pontifícia Universidade Católica do Paraná, Curitiba.

OpenStreetMap. (2020). Cidade Joinville, Região Geográfica Imediata de Joinville, Santa Catarina, Região Sul, Brasil. Recuperado em 1 de junho de 2020, de https://www.openstreetmap.org/relation/296650

Pinto, M. C. (2017). Waze e centros de comando e controle urbanos: estudos de casos no Brasil (Dissertação de mestrado). Programa de Pós-Graduação em Comunicação e Cultura Contemporâneas, Universidade Federal da Bahia, Salvador.

Prefeitura de Joinville. (2019). Smart Mobility: Cidades Inteligentes, Dados abertos de mobilidade. Apresentação da Secretaria de Planejamento Urbano e Desenvolvimento Sustentável para o Prêmio Smart City Business 2019. Joinville: Prefeitura de Joinville.

Rede Brasileira de Cidades Inteligentes e Humanas - RBCIH. (2020). o que são a Rede CHICS e o IBRACHICS?. Brasília: RBCIH. Recuperado em 1 de junho de 2020, de http://redebrasileira.org/institucional

Schiavo, M. (2019). Prêmio InovaCidade. São Paulo: Instituto Smart City Business America. Recuperado em 10 de dezembro de 2019, de http://www.expo.scbamerica.com/site/scbbr2019/premio-inovacidade

Vicente, V. F. (2017). Dados da multidão: análise da parceria entre Waze e prefeitura do Rio De Janeiro. In $5^{\circ}$ Simpósio Internacional LAVITS / Vigilancia, Democracia y Privacidad en América Latina: Vulnerabilidades y resistencias (Vol 5, p. 479499). Santiago, Chile: LAVTIS.

Waze. (2019). Conheça o case de Joinville do Waze For Cities Data. Recuperado em 8 de dezembro de 2019, de https://www.youtube.com/watch?v=xIhccla6onk

Wazeopedia. (2019). Wazeopedia. Recuperado em 8 de dezembro de 2019, de https://wazeopedia.waze.com/

Editor responsável: Fábio Duarte

Recebido: 31 jan. 2020

Aprovado: 23 dez. 2020 